\title{
Childhood dietary patterns and body composition at age 6 years: the Children of Screening for Pregnancy Endpoints (SCOPE) study
}

\author{
Angela C. Flynn ${ }^{1 *}$, John M. D. Thompson ${ }^{2,3}$, Kathryn V. Dalrymple ${ }^{1}$ Clare Wall $^{4}$, Shahina Begum ${ }^{1}$,
} Jaijus Pallippadan Johny ${ }^{3}$, Wayne S. Cutfield ${ }^{5,6}$, Robyn North ${ }^{5,6}$, Lesley M. E. McCowan ${ }^{3}$, Keith M. Godfrey ${ }^{7}$, Edwin A. Mitchell ${ }^{2}$ and Lucilla Poston ${ }^{1}$ on behalf of the SCOPE Consortium

${ }^{1}$ Department of Women and Children's Health, School of Life Course Sciences, King's College London, London, UK

${ }^{2}$ Department of Paediatrics, Child \& Youth Health, Faculty of Medical and Health Sciences, University of

Auckland, Auckland, New Zealand

${ }^{3}$ Department of Obstetrics and Gynaecology, Faculty of Medical and Health Sciences, University of Auckland, Auckland, New Zealand

${ }^{4}$ Department of Nutrition, School of Medical Sciences, University of Auckland, Auckland, New Zealand

${ }^{5}$ Liggins Institute, University of Auckland, Auckland, New Zealand

${ }^{6}$ Department of General Medicine, Auckland City Hospital, Auckland, New Zealand

${ }^{7}$ MRC Lifecourse Epidemiology Unit and NIHR Southampton Biomedical Research Centre, University of Southampton and University Hospital Southampton NHS Foundation Trust, Southampton, UK

(Submitted 27 September 2019 - Final revision received 25 January 2020 - Accepted 11 February 2020 - First published online 26 February 2020 )

\section{Abstract}

Dietary patterns describe the combination of foods and beverages in a diet and the frequency of habitual consumption. Better understanding of childhood dietary patterns and antenatal influences could inform intervention strategies to prevent childhood obesity. We derived empirical dietary patterns in 1142 children (average age 6.0 (SD 0.2) years) in New Zealand, whose mothers had participated in the Screening for Pregnancy Endpoints (SCOPE) cohort study and explored associations with measures of body composition. Participants (Children of SCOPE) had their diet assessed by FFQ, and dietary patterns were extracted using factor analysis. Three distinct dietary patterns were identified: 'Healthy', 'Traditional' and 'Junk'. Associations between dietary patterns and measures of childhood body composition (waist, hip, arm circumferences, BMI, bioelectrical impedance analysis-derived body fat \% and sum of skinfold thicknesses (SST)) were assessed by linear regression, with adjustment for maternal influences. Children who had higher 'Junk' dietary pattern scores had 0.24 (sD 0.08; $95 \%$ CI 0.04, 0.13) cm greater arm and 0.44 (sD 0.05; $95 \%$ CI $0 \cdot 01,0 \cdot 10) \mathrm{cm}$ greater hip circumferences and $1 \cdot 13$ (SD 0.07; $95 \% \mathrm{CI} 0 \cdot 03,0 \cdot 12$ ) $\mathrm{cm}$ greater SST and were more likely to be obese (OR $1 \cdot 74$; $95 \%$ CI 1.07, 2.82); those with higher 'Healthy' pattern scores were less likely to be obese (OR 0.62; $95 \%$ CI 0.39, 1.00). In a large mother-child cohort, a dietary pattern characterised by high-sugar and -fat foods was associated with greater adiposity and obesity risk in children aged 6 years, while a 'Healthy' dietary pattern offered some protection against obesity. Targeting unhealthy dietary patterns could inform public health strategies to reduce the prevalence of childhood obesity.

Key words: Dietary patterns: Body composition: Pregnancy: Childhood obesity

Childhood obesity impacts on both health in childhood and adult life $^{(1)}$. Global estimates from the WHO indicate that $18 \%$ of children and adolescents aged 5-19 years were overweight or obese in $2016^{(2)}$. Diet is a key determinant of childhood obesity ${ }^{(3)}$; as dietary habits established in early childhood may track into adulthood $^{(4,5)}$, interventions which change dietary intake early in life have the potential to improve lifelong health. Effective intervention strategies will depend on better understanding of the specific dietary elements which contribute to obesity risk.
The assessment of dietary patterns provides a practical alternative to the more conventional measurement of isolated nutrients and dietary components, the limitations of which are appreciated $^{(6)}$. Dietary patterns can be derived by one of the two approaches: theoretically, in which dietary variables are grouped according to a priori criteria of nutritional health, for example, a dietary index, or empirically, where dietary variables are condensed into clusters of variables using statistical modelling and examined $a$ posteriori ${ }^{(7)}$. This study used the empirical

Abbreviations: CoS, Children of SCOPE; SCOPE, Screening for Pregnancy Endpoints.

* Corresponding author: Dr Angela C. Flynn, email angela.flynn@kcl.ac.uk 
method as an assessment of total diet as it is more likely to be informative when addressing dietary risk factors for childhood obesity than diet indexes which focus on selected foods or nutrients.

Despite being widely explored in adult cohorts ${ }^{(8)}$, relationships between empirical dietary patterns and childhood obesity risk have seldom been studied ${ }^{(9,10)}$, especially in the context of contemporary mother-child cohorts. Ideally, associations between childhood dietary patterns and obesity are best examined in studies with information on the potentially confounding effects of maternal obesity, gestational diabetes and the mother's own dietary patterns ${ }^{(11,12)}$ and have potential to inform the development of complex interventions for mother and child.

Here, we describe a study in which we have determined empirical dietary patterns of 6-year-old children from the Children of Screening for Pregnancy Endpoints (SCOPE) (COS) study. Their mothers were participants in the Auckland New Zealand centre of the International SCOPE study ${ }^{(13)}$. The overall objective of $\mathrm{CoS}$ was to identify the early life modifiable determinants of obesity and insulin resistance in children at 5-6 years of age. We have examined relationships between dietary patterns and measures of childhood obesity as assessed by childhood body composition (waist, hip and arm circumferences; BMI; bioelectrical impedance analysis-derived body fat $\%$ and sum of skinfold thicknesses) with the aim of informing new targets for intervention. Associations of maternal influences on childhood dietary patterns were also examined and taken into account as potential confounders for associations between childhood diet and adiposity.

\section{Methods \\ Children of Screening for Pregnancy Endpoints (SCOPE)}

$\mathrm{CoS}$ is a follow-up study of the offspring of women who participated in the Auckland arm of the SCOPE cohort study. SCOPE is a prospective international cohort study which aimed to identify factors in early pregnancy that predicted women at high risk of developing pre-eclampsia, spontaneous preterm birth and fetal growth restriction ${ }^{(13)}$. Nulliparous women with a singleton pregnancy were recruited at 15 weeks' gestation and a second research visit carried out at 20 weeks' gestation; detailed demographic and clinical data were recorded at each visit and at delivery and for any pregnancy complications. Details of the SCOPE study have been previously described ${ }^{(13)}$. For a child to be eligible to participate in $\mathrm{CoS}$, the mother agreed to be contacted about the follow-up study and the child was aged between 5 and $6 \cdot 25$ years. Children with major health problems that might affect growth were excluded.

From the 2032 mothers included in the Auckland SCOPE cohort, 1208 (59\%) mothers and their children were recruited. We previously reported no difference in maternal BMI between participants in CoS and the original complete Auckland cohort; however, the mothers in CoS were more likely to be older, White, have a higher educational attainment and higher socioeconomic status, were less likely to smoke in their first trimester and were less likely to have delivered before 37 weeks $^{(14)}$. Of the 1208 mother-child dyads, thirty-five were excluded due to missing or implausible body fat \%. The final study population included 1173 mother-child dyads, and a total of 1142 children had information on dietary intake collected to extract dietary patterns. All women provided consent for participation of their children. Ethical approval was granted by the Auckland Ethics Committee (AKX/02/00/364).

\section{Dietary assessment and dietary patterns}

At the follow-up visit, a research nurse asked the child's main caregiver to complete a FFQ about the child's diet. This was based on a FFQ developed for the New Zealand Children's National Nutrition Survey ${ }^{(15)}$ and requests information on the consumption of 118 food and beverage items. Accompanying this list was a multiple response grid which estimated frequency of consumption of the food and beverages in the preceding month, ranging from 'never or less than once per month' to 'two or more times per d'. Each food and beverage item had a standard serving size (e.g. slice of bread, apple). Where individual food items were unanswered, we imputed data using the MICE methodology. We used ten burn-ins, followed by the construction of ten imputed data sets. Data were imputed if $<20 \%$ of items were missing.

Factor analysis with varimax rotation was performed to derive dietary patterns in each of the imputed data sets. The number of factors was chosen using the scree plot of eigenvalues and the interpretability of the factor loadings. Foods with a factor loading of $\geq \pm 0.30$ were considered to have a strong association with that factor. We then averaged factor loading for the dietary patterns across the imputed data set. These factor loadings were then applied across an averaged imputed data set constructed by averaging the responses across the imputed data sets. Sensitivity analyses were carried out to assess the MICE methodology to simple imputation of making an assumption that missed items were not answered because they were not consumed.

\section{Childhood body composition}

At the follow-up visit, the child's body composition was assessed using anthropometric and bioelectrical impedance analysis measurements. Arm circumference $(\mathrm{cm})$ was measured at the mid-point between the edge of the shoulder bone and bony tip of the right elbow. Waist circumference $(\mathrm{cm})$ was assessed at the mid-point between the lowest rib and top of the hip. Hip circumference $(\mathrm{cm})$ was taken as the distance around the maximal diameter of buttocks. For estimation of BMI $\left(\mathrm{kg} / \mathrm{m}^{2}\right)$, weight $(\mathrm{kg})$ was measured in light clothing without shoes to the nearest $0 \cdot 1 \mathrm{~kg}$ and height $(\mathrm{cm})$ assessed in a standing position with a stadiometer. Childhood obesity was defined as BMI $z$-score $>2$ SD according to WHO's child growth standards ${ }^{(16)}$. Body fat $\%$ was determined by bioelectrical impedance analysis (ImpediMed SFB7 tetra polar bioimpedance spectroscopy). Fat-free mass was calculated using: fat-free mass $=0.65$ (height ${ }^{2} /$ impedance) $+0 \cdot 686 \times$ age $+0 \cdot 15^{(17)}$. Body fat $\%$ was estimated using: (weight - fat-free mass)/weight $\times 100$. Subscapular, triceps, biceps and suprailiac skinfold thicknesses $(\mathrm{mm})$ were measured using Holtain skinfold callipers and summed to give sum of skinfold thicknesses. All measurements were taken in triplicate and mean values calculated. 


\section{Covariates}

In the SCOPE study, maternal demographic, clinical and lifestyle data were recorded. This included maternal age, BMI $\left(\mathrm{kg} / \mathrm{m}^{2}\right)$ at 15 weeks' gestation, ethnicity, socio-economic status (New Zealand Socio-economic Index 1996, education (years)), alcohol use pre-pregnancy, smoking during the first trimester, multivitamin use pre-pregnancy, maternal mood (Edinburgh Postnatal Depression Scale at 15-week visit), television (number of hours watched per $\mathrm{d}$ in the last month, evaluated at the 15-week visit), gestational hypertension (defined as systolic blood pressure $\geq 140 \mathrm{mmHg}$ or diastolic blood pressure $\geq 90 \mathrm{mmHg}$ on at least two occasions after the 20 -week visit and before onset of labour), gestational diabetes mellitus diagnosis (any of: (1) $75 \mathrm{~g}$ oral glucose tolerance test (OGTT) fasting glucose $\geq 5.5 \mathrm{mmol} / \mathrm{l}$ and/or $2 \mathrm{~h} \geq 9.0 \mathrm{mmol} / \mathrm{l}$, or (2) polycose $1 \mathrm{~h}$ glucose $>11.0$ $\mathrm{mmol} / \mathrm{l}$ and no OGTT available, or (3) fasting glucose $\geq 5.5 \mathrm{mmol} / \mathrm{l}$ and no polycose or OGTT available, or (4) random glucose $>11 \mathrm{mmol} / \mathrm{l}$ and no polycose, OGTT or fasting glucose), biomarkers measured from maternal blood samples including placental growth factor. At the time of birth, data were collected on premature birth, birth weight (g), gestational age (weeks), child's age and sex and breast-feeding (exclusive breast-feeding at discharge form obstetric unit).

\section{Statistical analysis}

Descriptive data are presented as mean values and standard deviations, frequencies and percentages, or medians and interquartile ranges as appropriate. Multiple regression analysis was carried out to examine the associations between maternal influences (including maternal BMI, ethnicity, cigarette smoking in the first trimester, multivitamin use pre-pregnancy, breastfeeding, socio-economic status, education and age ${ }^{(12,18)}$ ) and children's dietary patterns. To investigate the relationship between childhood dietary patterns and body composition, linear regression was used. Two models were generated: model 1 , where simple linear regression with no adjustment for confounding variables was performed, and model 2 , multiple linear regression including adjustment for confounders. Confounders were chosen a priori based on known associations with obesity ${ }^{(14,19-29)}$. The maternal and early postnatal exposures used in the adjusted model were maternal BMI, ethnicity, socioeconomic status, multivitamin use pre-pregnancy, alcohol intake pre-pregnancy, cigarette smoking in first trimester, time spent watching television in first trimester, depression in first trimester, gestational hypertension, gestational diabetes mellitus diagnosis, maternal plasma placental growth factor at 15 weeks' gestation, breast-feeding and infant sex. Standardised $\beta$-coefficients are presented which represent the SD in body composition measure per SD change in dietary pattern score, enabling comparison across outcomes. Data were analysed using Stata software, version 15.0 (StataCorp).

\section{Results}

\section{Characteristics of the study population}

The characteristics of the women and children are presented in Table 1. At 15 weeks' gestation, the average age of the
Table 1. Maternal and child characteristics in Screening for Pregnancy Endpoints (SCOPE) and the Children of SCOPE studies ( $n$ 1142) (Mean values and standard deviations; numbers and percentages; median values and interquartile ranges)

\begin{tabular}{|c|c|c|c|}
\hline Maternal characteristics (15 weeks' gestation) & $n$ & & $\%$ \\
\hline \multicolumn{4}{|l|}{ Age (years) } \\
\hline Mean & \multicolumn{3}{|c|}{$31 \cdot 0$} \\
\hline SD & \multicolumn{3}{|c|}{4.4} \\
\hline \multicolumn{4}{|l|}{ BMI $\left(\mathrm{kg} / \mathrm{m}^{2}\right)$} \\
\hline Median & \multicolumn{3}{|c|}{23.9} \\
\hline Interquartile range & \multicolumn{3}{|c|}{$21 \cdot 8-26 \cdot 6$} \\
\hline \multicolumn{4}{|l|}{ BMI (categorical; kg/m²) } \\
\hline$<18.5$ & 8 & & 0.7 \\
\hline $18 \cdot 5-24 \cdot 9$ & 698 & & 61.2 \\
\hline $25 \cdot 0-29 \cdot 9$ & 321 & & $28 \cdot 1$ \\
\hline$\geq 30$ & 113 & & 9.9 \\
\hline \multicolumn{4}{|l|}{ Ethnicity } \\
\hline White & 1001 & & $87 \cdot 6$ \\
\hline Asian & 44 & & 3.9 \\
\hline Indian & 36 & & $3 \cdot 2$ \\
\hline Maori & 34 & & 2.9 \\
\hline Other & 16 & & 1.4 \\
\hline Pacific & 11 & & 0.9 \\
\hline \multicolumn{4}{|l|}{ Socio-economic status (NZSEI) } \\
\hline High (56-90) & 315 & & $27 \cdot 6$ \\
\hline Medium (32-55) & 677 & & $59 \cdot 3$ \\
\hline Low $(10-31)$ & 150 & & $13 \cdot 1$ \\
\hline \multicolumn{4}{|l|}{ Education (years) } \\
\hline$<12$ & 65 & & $5 \cdot 7$ \\
\hline $12-13$ & 1058 & & $92 \cdot 6$ \\
\hline$>13$ & 19 & & 1.6 \\
\hline Smoking in first trimester & 107 & & $9 \cdot 4$ \\
\hline Alcohol intake pre-pregnancy & 631 & & 54.5 \\
\hline Multivitamin use pre-pregnancy & 377 & & 33.3 \\
\hline Gestational diabetes & 18 & & 1.7 \\
\hline Gestational hypertension & 61 & & $5 \cdot 3$ \\
\hline \multicolumn{4}{|l|}{ Television (hours watched per d) } \\
\hline$<2$ & 483 & & $42 \cdot 4$ \\
\hline $2-4$ & 586 & & 51.5 \\
\hline$>4$ & 70 & & $6 \cdot 2$ \\
\hline \multicolumn{4}{|l|}{ Characteristics at follow-up } \\
\hline \multicolumn{4}{|l|}{ Maternal BMI (categorical; kg/m²) } \\
\hline$<18.5$ & 23 & & $2 \cdot 0$ \\
\hline $18 \cdot 5-24.9$ & 622 & & 54.5 \\
\hline $25 \cdot 0-29 \cdot 9$ & 327 & & $28 \cdot 7$ \\
\hline$\geq 30$ & 169 & & 14.8 \\
\hline \multicolumn{4}{|l|}{ Child's age } \\
\hline Mean & & $6 \cdot 0$ & \\
\hline SD & & 0.2 & \\
\hline Child's sex (female) & 578 & & $50 \cdot 6$ \\
\hline Child's height $(\mathrm{cm})$ & & & \\
\hline Mean & & $117 \cdot 9$ & \\
\hline $\mathrm{SD}$ & & 4.9 & \\
\hline Child's weight $(\mathrm{kg})$ & & & \\
\hline Median & & $21 \cdot 7$ & \\
\hline Interquartile range & & $20 \cdot 0-23 \cdot 7$ & \\
\hline Child's BMI (z-score) & & & \\
\hline Mean & & $0 \cdot 28$ & \\
\hline SD & & 0.91 & \\
\hline Child overweight or obese (BMl $z$-score $\geq 1$ & $\mathrm{SD})^{*}$ & & \\
\hline Mean & & 176 & \\
\hline $\mathrm{SD}$ & & $15 \cdot 4$ & \\
\hline Child overweight (BMI $z$-score $\geq 1 \mathrm{sD},<2 \mathrm{SD}$ ) & & & \\
\hline Mean & & 145 & \\
\hline SD & & $12 \cdot 7$ & \\
\hline Child obese (BMI $z$-score $\geq 2$ sD) & & & \\
\hline Mean & & 31 & \\
\hline SD & & 2.71 & \\
\hline Child's body fat \% & & & \\
\hline Mean & & $22 \cdot 8$ & \\
\hline SD & & $6 \cdot 3$ & \\
\hline
\end{tabular}


Table 1. (Continued)

\begin{tabular}{lr}
\hline Maternal characteristics (15 weeks' gestation) & \\
\hline Child's arm circumference (cm) & \\
Mean & 18.6 \\
SD & 1.7 \\
Child's waist circumference (cm) & \\
Mean & 55.4 \\
SD & 4.0 \\
Child's hip circumference (cm) & \\
Mean & 61.6 \\
SD & 4.9 \\
Child's sum of skinfolds (mm) & 30.5 \\
Mean & 9.3 \\
SD &
\end{tabular}

NZSEI, New Zealand Socio-Economic Index.

* World Health Organization $(2007)^{(16)}$.

mothers was 31.0 ( $\mathrm{SD} 4.4$ ) years and the median BMI was 23.9 (interquartile range $21 \cdot 8-26.6) \mathrm{kg} / \mathrm{m}^{2}$. The majority of women were of White ethnicity $(87.6 \%)$. The average age of the children at the follow-up visit was $6 \cdot 0$ (SD 0.2) years. A total of $176(15.1 \%)$ children were overweight or obese according to WHO standards.

\section{Dietary patterns}

A total of 1142 children had complete data in the imputed data sets. Factor analysis identified three distinct dietary patterns at age 6 years; for ease of description, we have termed these as Healthy, Traditional and Junk in line with other previously published studies ${ }^{(30)}$. The factor loadings for the dietary patterns are shown in online Supplementary Table S1. The 'Healthy' diet was characterised by high loadings including a number of fruits (berries, stone fruits, feijoas/kiwifruit, pineapple) and vegetables (avocado, tomato, green leafy vegetables, celery, cucumber, green beans, pepper). The 'Traditional' diet was based on a number of meat (meat and poultry in casseroles, roasted as part of a dish) and carbohydrate (potato, taro, kumara, and pumpkin either steamed, boiled or roasted). The 'Junk' diet was characterised by high loadings on candy bars, potato crisps, lollies or sweets, sausage rolls, and bought cake or muffin (Table 2). A number of other items in the Junk dietary pattern had loadings between 0.25 and 0.30 but were not consistently above 0.3 across factor analyses of the imputed data sets.

In sensitivity analyses, a factor analysis using data imputed by replacing missing data with never resulted in very similar dietary patterns and loadings. Examination of the imputed data set showed that in general, a large proportion of missing values on individual items were imputed as never.

\section{Associations between maternal influences and dietary patterns}

The dietary patterns had differing associations with maternal influences, which were robust to adjustment (Table 3). Children who had higher 'Healthy' dietary pattern scores were more likely to have mothers who had a lower early pregnancy BMI, of Indian ethnicity (and less likely to be Maori), who had a higher level of educational attainment and who took multivitamins pre-pregnancy (all $P<0 \cdot 05$ ). Children who had higher
Table 2. Dietary patterns identified using factor analysis

\begin{tabular}{|c|c|c|c|}
\hline Variable & Healthy & Traditional & Junk \\
\hline Avocado & $0.45^{\star}$ & 0.03 & -0.09 \\
\hline Green beans & $0.34^{*}$ & 0.15 & -0.07 \\
\hline Berries & $0.49^{*}$ & $-0 \cdot 13$ & -0.02 \\
\hline Cake/muffin/bun & 0.15 & 0.05 & $0.32^{*}$ \\
\hline Brown rice & $0.36^{*}$ & 0.03 & -0.09 \\
\hline Candy bars & 0.05 & 0.10 & $0.46^{*}$ \\
\hline Celery & $0.38^{*}$ & 0.11 & 0.01 \\
\hline Cucumber & $0.34^{*}$ & -0.06 & -0.03 \\
\hline Dried fruit & $0.32^{*}$ & 0.06 & -0.02 \\
\hline Feijoas/kiwifruit & $0.38^{*}$ & 0.12 & 0.02 \\
\hline Fish fillets not in breadcrumb/batter & $0.31^{*}$ & 0.11 & -0.03 \\
\hline Green leafy vegetables & $0.41^{*}$ & 0.25 & -0.08 \\
\hline Kumera/pumpkin roasted & 0.18 & $0.42^{*}$ & 0.01 \\
\hline Kumera/pumpkin steamed or boiled & 0.19 & $0.37^{*}$ & -0.03 \\
\hline Lentils/chickpeas/other beans & $0.37^{*}$ & 0.09 & $-0 \cdot 12$ \\
\hline Lollies/sweets & -0.03 & -0.09 & $0.31^{*}$ \\
\hline Meat as part of dish (beef/pork/lamb) & 0.05 & $0.36^{*}$ & 0.02 \\
\hline Meat casseroles (beef, pork, lamb) & 0.08 & $0.45^{\star}$ & 0.04 \\
\hline Meat roasted (beef/pork/lamb) & 0.02 & $0.42^{*}$ & 0.12 \\
\hline Mixed vegetables & 0.18 & $0.40^{*}$ & 0.09 \\
\hline Nuts & $0.33^{*}$ & -0.01 & -0.02 \\
\hline Other fruit & $0.33^{*}$ & 0.11 & -0.02 \\
\hline Pepper & $0.34^{*}$ & 0.05 & -0.05 \\
\hline Pineapple & $0 \cdot 37^{\star}$ & 0.09 & 0.08 \\
\hline Potato/other crisps & -0.17 & 0.06 & $0.34^{*}$ \\
\hline Potato/taro roasted & 0.09 & $0.34^{*}$ & 0.08 \\
\hline Potato/taro steamed or boiled & 0.03 & $0.36^{\star}$ & 0.01 \\
\hline Poultry as part of dish & 0.11 & $0.38^{*}$ & 0.02 \\
\hline Poultry casseroles & 0.15 & $0.44^{*}$ & 0.07 \\
\hline Poultry roasted & 0.01 & $0.32^{*}$ & 0.11 \\
\hline Sausage rolls & 0.07 & 0.19 & $0.30^{*}$ \\
\hline Stone fruit & $0.45^{\star}$ & -0.06 & 0.01 \\
\hline Tomato & $0.43^{*}$ & 0.00 & -0.03 \\
\hline Yogurt & $0.31^{*}$ & 0.02 & -0.01 \\
\hline
\end{tabular}

* Factor loadings $\geq \pm 0.3$ for each identified dietary pattern.

'Traditional' dietary pattern scores were more likely to have mothers of Pacific Islander ethnicity, who had a lower socioeconomic status were younger (all $P<0 \cdot 05$ ). Children who had higher 'Junk' dietary pattern scores were more likely to have mothers of Indian or Pacific Islander ethnicity that smoked in the first trimester and had a lower educational attainment (all $P<0.05$ ).

\section{Associations between children's dietary patterns and body composition}

The association between children's dietary patterns and body composition at age 6 years is shown in Table 4. Children who had higher 'Healthy' pattern scores were less likely to be obese according to WHO child growth standards in models 1 and 2 (adjusted OR 0.62; $95 \%$ CI 0.39, 1.00).

Children who had higher 'Junk' pattern scores had greater arm (adjusted $\beta=0 \cdot 08 ; 95 \%$ CI $0 \cdot 04,0 \cdot 13$ ) and hip (adjusted $\beta=0.05 ; 95 \%$ CI 0.01, 0.10) circumferences and sum of skinfold thicknesses (adjusted $\beta=0 \cdot 07 ; 95 \%$ CI 0.03, 0.12). Furthermore, children who had higher 'Junk' pattern scores were more likely to be obese (OR 1.74; $95 \%$ CI 1.07, 2·82). These associations were robust to adjustments for confounders.

Children who had higher 'Traditional' dietary pattern scores had greater arm, waist and hip circumferences, body fat $\%$ and 
BMI in the univariable analysis. These associations were not robust to adjustments for confounders.

\section{Discussion}

In this large contemporary mother and child cohort, we found one distinct empirical childhood dietary pattern to be associated with greater adiposity and obesity risk in children aged 6 years. This pattern was characterised by high intakes of foods and beverages high in sugar and/or fat including saturated fat. A healthy dietary pattern characterised by a number of fruits and vegetables was associated with a reduced risk of obesity in a small number of children. Furthermore, we were able to characterise influences of maternal antenatal exposures on childhood dietary patterns which included maternal ethnicity, age, BMI, education, socio-economic status and smoking in pregnancy.

The majority of reports of childhood dietary patterns have addressed the hypothesis that dietary behaviours in early childhood can influence the risk of obesity in later childhood. Several studies have shown a strong relationship between unhealthy dietary patterns characterised by energy dense, low-fibre and high-fat foods and their later risk of obesity ${ }^{(31,32)}$. Few studies have examined the relationship between empirical dietary patterns and childhood body composition measured simultaneously. In one of the few to have specifically addressed this association, the Lifeways Cross-Generational Cohort study in Irish children aged 5 years showed that the upper tertiles of a 'cereal and juice' dietary pattern and the middle tertile of a 'pasta and veg' dietary pattern were negatively associated with overweight and obesity ${ }^{(33)}$. Our findings together with observations that diet in early childhood is a risk for later obesity may reflect unchanging dietary patterns throughout early and midchildhood $^{(4)}$, suggesting that interventions which specifically target foods and snacks high in sugar and fat in early childhood could play a role in reducing later risk of obesity. The observation that the relationship between the Junk and Healthy patterns and BMI observed in this study was only evident in children classified as obese is particularly relevant to public health measures for the prevention of childhood obesity.

The three dietary patterns identified in this study ('Traditional', 'Junk' and 'Healthy') are consistent with those described in the New Zealand birth cohort study which assessed dietary patterns in Auckland children aged 3.5 and 7 years ${ }^{(30)}$. Similarities were observed for high factor loadings for the 'traditional' diet of meat, vegetables and potatoes, the 'healthy' diet of fruit, vegetables and nuts, and the junk diet of confectionery.

We identified important associations between maternal influences and childhood dietary patterns. Better quality diets were more common in children who had mothers with higher educational attainment, and who had a lower BMI, similar to observations in the Southampton Women's Survey ${ }^{(12,34)}$. Consistent with ALSPAC data, poorer diets were more common in children who were born to mothers who had lower educational attainment ${ }^{(18)}$. Furthermore, the mothers of children with poorer diets were more likely to smoke in pregnancy, which is similar to the New Zealand birth cohort study ${ }^{(30)}$. Recently, we 
Table 4. Multiple regression analysis of the dietary patterns associated with body composition at age 6 years* (Standardised $\beta$-coefficients and odds ratios and $95 \%$ confidence intervals)

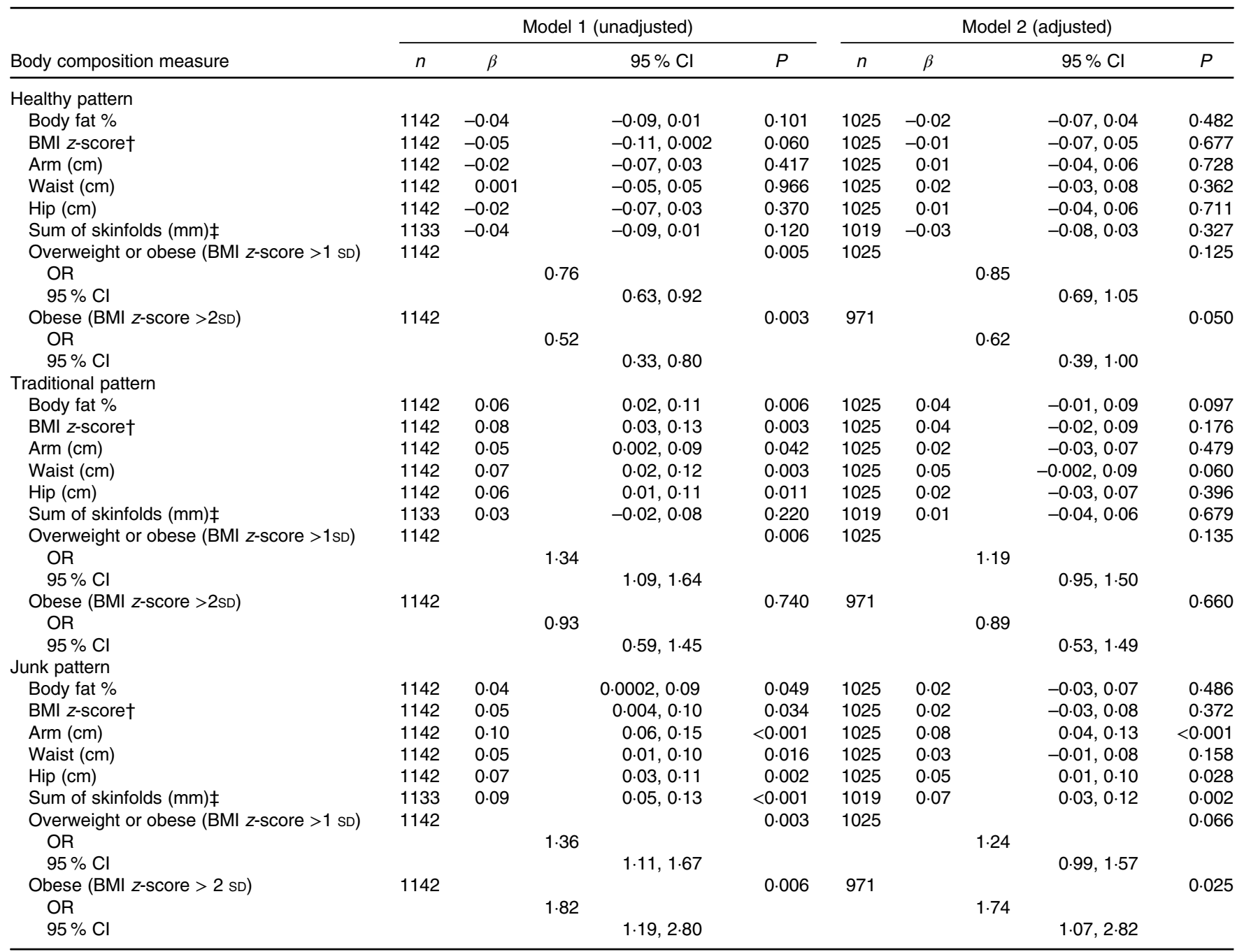

* Standardised coefficient $(\beta)$, representing the standard deviation (SD) change and $95 \% \mathrm{Cl}$ in body composition measure per SD change in dietary pattern score enabling comparison across outcomes; model 1 (simple regression): association between dietary patterns and childhood body composition at age 6 years; model 2 (multiple regression): association between dietary patterns and childhood body composition, adjusting for infant mode of feeding, infant sex, and maternal ethnicity, BMl, socio-economic status, multivitamin use prepregnancy, alcohol intake pre-pregnancy, cigarette smoking in first trimester, time spent watching television in first trimester, depression in first trimester, gestational hypertension, gestational diabetes mellitus diagnosis and plasma placental growth factor.

† World Health Organization (2007) ${ }^{(16)}$ BMI for age $z$-score.

$\ddagger$ Sum of triceps, biceps, subscapular and suprailiac skinfold thicknesses.

reported on the relationship between maternal BMI and childhood obesity risk in the CoS cohort. Maternal BMI was independently associated with childhood BMI $z$-score, sum of skinfolds and body fat $\%(\text { all } P<0.001)^{(14)}$. The global increase in obesity, which is reflected in women who present for antenatal care has led to the concern that obesity in pregnancy may contribute to childhood obesity risk, with evidence from observational studies to support this link ${ }^{(35)}$. Exposure in utero to the metabolic consequences of a high maternal BMI including dyslipidaemia, inflammatory markers, hyperinsulinaemia and hyperleptinaemia appears to influence offspring obesity risk $^{(36,37)}$. This highlights the need for public health interventions to promote a healthy BMI and better quality diet in reproductive aged women.
Strengths of the present study include this being an addition to a very sparse literature which has defined the dietary patterns associated with childhood obesity. Whilst the assumption is often made that foods and snacks high in sugar and fat are major contributors, there is limited evidence to support this widely held assumption in paediatric cohorts. Furthermore, our observations have been made in a large cohort, compared with previous studies of childhood obesity in mother-child cohorts. By focusing on obesity in the children, the CoS protocol prospectively included detailed measurements of childhood adiposity and a comprehensive data set to examine maternal influences on childhood diet. By characterising dietary patterns, we have been able to capture the complexity of the diet while overcoming the limitations of single nutrient assessment ${ }^{(6)}$. 
Whilst this study provides evidence that specific dietary patterns in children are linked to childhood obesity, there are some limitations. At follow-up, data from 1208 (59\%) of the 2032 mothers and their children from the original Auckland arm of the SCOPE cohort were available, which could lead to erroneous conclusions if the relationships between dietary patterns and childhood body composition differ between those included in follow-up and those not. Being observational in design, we are only able to examine associations and not infer causation between dietary patterns and childhood obesity development. We assessed dietary intake using an FFQ, which may be prone to bias ${ }^{(38)}$. Factor analysis was used to derive empirical dietary patterns which involves several subjective decisions including the number of factors to extract, rotation method and naming of the factors ${ }^{(39)}$. We adjusted for several potentially confounding variables in the assessment of the relationship between maternal influences and childhood dietary patterns and between childhood dietary patterns and obesity. However, the possibility of residual confounding due to unmeasured lifestyle factors cannot be excluded. The relationship between physical activity and inactivity was not assessed in the present study. WHO guidelines on childhood obesity prevention include promoting physical activity among children in the first few years of life in addition to focusing on dietary intake ${ }^{(40)}$. Evidence from cross-sectional studies examining the association between physical activity in children and adiposity suggest that higher levels of physical activity are related to reduced adiposity ${ }^{(41)}$. Associations with sedentary time are less consistent ${ }^{(42)}$. Future studies should include physical activity in assessing the relationship between childhood health behaviours and obesity risk. Finally, the majority of women in this study were of European descent and were well educated and were not a representative cohort of New Zealand women and children.

\section{Conclusion}

This mother-child cohort study with multiple measures of childhood body composition provides support for a relationship between empirical dietary patterns representing overall diet and childhood adiposity. A dietary pattern characterised by consumption of high sugar and fat including saturated fat foods was associated with greater body circumferences, sum of skinfolds and obesity risk in children aged 6 years, while a 'Healthy' dietary pattern offered some apparent protection against obesity. We also identified a number of maternal exposures that were associated with eating patterns in early childhood providing insights into important maternal influences on children's dietary intake. Our data add to the growing evidence that targeting maternal obesity and unhealthy dietary patterns associated with early childhood adiposity could inform public health strategies to reduce the prevalence of childhood obesity.

\section{Acknowledgements}

The authors are grateful to the women and children who participated in the Children of SCOPE study. The authors thank the SCOPE Project Manager Rennae Taylor and research assistants
(Noleen Van Zyl, Elin Granrud and Desley Minahan) for their contributions throughout the study.

K. M. G. is supported by the UK Medical Research Council (MC_UU_12011/4), the National Institute for Health Research (NIHR Senior Investigator (NF-SI-0515-10042), NIHR Southampton 1000DaysPlus Global Nutrition Research Group) and NIHR Southampton Biomedical Research Centre), the European Union (Erasmus+Programme Early Nutrition eAcademy Southeast Asia-573651-EPP-1-2016-1-DE-EPPKA2CBHE-JP), the US National Institute On Aging of the National Institutes of Health (grant no. U24AG047867) and the UK ESRC and BBSRC (grant no. ES/M00919X/1). L. P. is supported by the Biomedical Research Centre at Guy's and St. Thomas' NHS Foundation Trust and King's College London and Tommy's Charity. The funders had no role in study design, data collection and analysis, decision to publish or preparation of the manuscript. SCOPE funding: University of Auckland, the New Enterprise Research Fund, Foundation for Research Science and Technology, Health Research Council of New Zealand, Evelyn Bond Fund, Auckland District Health Board Charitable Trust. Children of SCOPE funding: Health Research Council of New Zealand and Cure Kids. E. A. M. and J. M. D. T. were supported by Cure Kids.

A. C. F., S. B., J. M. D. T., J. P. J. analysed and interpreted the data. E. A. M. directed the Children of Scope Study. C. W. and R. $\mathrm{N}$. were involved in data collection, investigation and analysis. A. C. F. and K. V. D. completed additional analyses for the data. A. C. F., K. V. D. and L. P. had overall responsibility for the manuscript. All authors have read and approved the final manuscript.

L. P. is part of an academic consortium that has received research funding from Abbott Nutrition and Danone. K. M. G. reports reimbursement from Nestle Nutrition Institute, has a patent Phenotype prediction issued, a patent Predictive use of CpG methylation issued, a patent Maternal Nutrition Composition pending, a patent Vitamin $\mathrm{B}_{6}$ in maternal administration for the prevention of overweight or obesity in the offspring issued and is part of an academic consortium that with W. S. C. has received research funding from Abbott Nutrition, Nestec and Danone. C. W. has received funding for an investigator initiated trial from Danone Nutricia. The other authors declare that there are no conflicts of interest.

\section{Supplementary material}

For supplementary material referred to in this article, please visit https://doi.org/10.1017/S0007114520000628

\section{References}

1. Reilly JJ \& Kelly J (2011) Long-term impact of overweight and obesity in childhood and adolescence on morbidity and premature mortality in adulthood: systematic review. Int J Obes $\mathbf{3 5}$, 891-898.

2. World Health Organization (2016) Report of the Commission on Ending Childhood Obesity. https://www.who.int/gho/ncd/ risk_factors/overweight_obesity/overweight_adolescents/ en/ (accessed March 2019). 
3. Lobstein T, Baur L \& Uauy R (2004) Obesity in children and young people: a crisis in public health. Obes Rev 5, 4-85.

4. Northstone K \& Emmett PM (2008) Are dietary patterns stable throughout early and mid-childhood? A birth cohort study. Br J Nutr 100, 1069-1076.

5. Mikkilä V, Räsänen L, Raitakari OT, et al. (2005) Consistent dietary patterns identified from childhood to adulthood: the cardiovascular risk in Young Finns Study. BrJ Nutr 93, 923-931.

6. Hu FB (2002) Dietary pattern analysis: a new direction in nutritional epidemiology. Curr Opin Lipidol 13, 3-9

7. Newby PK \& Tucker KL (2004) Empirically derived eating patterns using factor or cluster analysis: a review. Nutr Rev 62, 177-203.

8. Rezagholizadeh F, Djafarian K, Khosravi S, et al. (2017) A posteriori healthy dietary patterns may decrease the risk of central obesity: findings from a systematic review and meta-analysis. Nutr Res 41, 1-13.

9. Ambrosini GL (2014) Childhood dietary patterns and later obesity: a review of the evidence. Proc Nutr Soc 73, 137-146.

10. Rocha NP, Milagres LC, Longo GZ, et al. (2017) Association between dietary pattern and cardiometabolic risk in children and adolescents: a systematic review. J Pediatr 93, 214-222.

11. Logan KM, Gale C, Hyde MJ, et al. (2017) Diabetes in pregnancy and infant adiposity: systematic review and meta-analysis. Arch Dis Child Fetal Neonatal Ed 102, F65-F72.

12. Fisk CM, Crozier SR, Inskip HM, et al. (2011) Influences on the quality of young children's diets: the importance of maternal food choices. Br J Nutr 105, 287-296.

13. Kenny LC, Black MA, Poston L, et al. (2014) Early pregnancy prediction of preeclampsia in nulliparous women, combining clinical risk and biomarkers: the Screening for Pregnancy Endpoints (SCOPE) international cohort study. Hypertension 64, 644-652.

14. Dalrymple KV, Begum S, Thompson JMD et al. (2019) Relationships of maternal body mass index and plasma biomarkers with childhood body mass index and adiposity at 6 years: the Children of SCOPE study. Paediatr Obes 14, e12537.

15. Metcalf PA, Scragg RKR, Sharpe S, et al. (2003) Short-term repeatability of a food frequency questionnaire in New Zealand children aged 1-14 y. Eur J Clin Nutr 57, 1498-1503.

16. World Health Organization (2008) Training Course on Child Growth Assessment: WHO Child Growth Standards. Geneva: WHO.

17. Schaefer F, Georgi M, Zieger A, et al. (1994) Usefulness of bioelectric impedance and skinfold measurements in predicting fat-free mass derived from total body potassium in children. Pediatr Res 35, 617-624

18. Northstone K \& Emmett P (2005) Multivariate analysis of diet in children at four and seven years of age and associations with socio-demographic characteristics. Eur J Clin Nutr 59, 751-760.

19. Power ML \& Schulkin J (2008) Sex differences in fat storage, fat metabolism, and the health risks from obesity: possible evolutionary origins. Br J Nutr 99, 931-940.

20. Taveras EM, Gillman MW, Kleinman K, et al. (2010) Racial/ ethnic differences in early-life risk factors for childhood obesity. Pediatrics 125, 686-995.

21. Falconer CL, Park MH, Croker H, et al. (2014) Can the relationship between ethnicity and obesity-related behaviours among school-aged children be explained by deprivation? A crosssectional study. BMJ Open $\mathbf{4}$, e003949.

22. Szeto IMY, Das PJ, Aziz A, et al. (2009) Multivitamin supplementation of Wistar rats during pregnancy accelerates the development of obesity in offspring fed an obesogenic diet. Int J Obes 33, 364-372.
23. Patel N, Dalrymple KV, Briley AL, et al. (2018) Mode of infant feeding, eating behaviour and anthropometry in infants at 6-months of age born to obese women - a secondary analysis of the UPBEAT trial. BMC Pregnancy Childbirth 18, 355.

24. Lampard AM, Franckle RL \& Davison KK (2014) Maternal depression and childhood obesity: a systematic review. Prev Med 59, 60-67.

25. Mizutani T, Suzuki K, Kondo N, et al. (2007) Association of maternal lifestyles including smoking during pregnancy with childhood obesity. Obesity 15, 3133-3139.

26. Leng J, Li W, Zhang S, et al. (2015) GDM women's prepregnancy overweight/obesity and gestational weight gain on offspring overweight status. PLOS ONE 10, e0129536.

27. Zheng JS, Liu H, Ong KK, et al. (2017) Maternal blood pressure rise during pregnancy and offspring obesity risk at 4 to 7 years old: the Jiaxing Birth Cohort. J Clin Endocrinol Metab 102, 4315-4322.

28. Strauss RS (1997) Effects of the intrauterine environment on childhood growth. Br Med Bull 53, 81-95.

29. Pervanidou P, Chouliaras G, Akalestos A, et al. (2014) Increased placental growth factor (PlGF) concentrations in children and adolescents with obesity and the metabolic syndrome. Hormones 13, 369-374.

30. Wall CR, Thompson JMD, Robinson E, et al. (2013) Dietary patterns of children at 3.5 and 7 years of age: a New Zealand birth cohort study. Acta Paediatr 102, 137-142.

31. Johnson L, Mander AP, Jones LR, et al. (2008) Energy-dense, low-fiber, high-fat dietary pattern is associated with increased fatness in childhood. Am J Clin Nutr 87, 846-854.

32. Ambrosini GL, Emmett PM, Northstone K, et al. (2012) Identification of a dietary pattern prospectively associated with increased adiposity during childhood and adolescence. Int $J$ Obes 36, 1299-1305.

33. Murrin CM, Heinen MM \& Kelleher CC (2015) Are dietary patterns of mothers during pregnancy related to children's weight status? Evidence from the lifeways cross-generational Cohort Study. AIMS Public Health 2, 274-296.

34. Okubo H, Crozier SR, Harvey NC, et al. (2015) Diet quality across early childhood and adiposity at 6 years: the Southampton Women's Survey. Int J Obes 39, 1456-1462.

35. Heslehurst N, Vieira R, Akhter Z, et al. (2019) The association between maternal body mass index and child obesity: a systematic review and meta-analysis. PLoS Med 16, e1002817.

36. Catalano PM, Farrell K, Thomas A, et al. (2009) Perinatal risk factors for childhood obesity and metabolic dysregulation. Am J Clin Nutr 90, 1303-1313.

37. Nelson SM, Matthews P \& Poston L (2009) Maternal metabolism and obesity: modifiable determinants of pregnancy outcome. Hum Reprod Update 16, 255-275.

38. Serdula MK, Alexander MP, Scanlon KS, et al. (2002) What are preschool children eating? A review of dietary assessment. Annu Rev Nutr 21, 475-498.

39. Martínez ME, Marshall JR \& Sechrest L (1998) Invited commentary: factor analysis and the search for objectivity. Am J Epidemiol 148, 17-19.

40. World Health Organization (2015) Interim report of the commission on ending childhood obesity. Geneva: WHO. https://www. who.int/end-childhood-obesity/publications/echo-report/en/ (accessed December 2019).

41. Jimenez-Pavon D \& Kelly JRJ (2010) Associations between objectively measured habitual physical activity and adiposity in children and adolescents: systematic review. Int J Pediatr Obes 5, 3-18.

42. Ekelund U, Hildebrand M \& Collings PJ (2014) Physical activity, sedentary time and adiposity during the first two decades of life. Proc Nutr Soc 73, 319-329. 